\title{
A Facial Expression Recognition Method for Baby Video Surveillance
}

\author{
Wenping $\mathrm{Lu}^{1}$, Min Li, Wenfei Hao and Yu Liu
}

\begin{abstract}
Facial expression recognition (FER) has been widely used in video surveillance and other fields. In this paper, we built a face image database containing four different kinds of infant expressions. Based on the analysis of the features of infants' eyes and mouths, this paper proposed a method of infant facial expression recognition. The experimental results showed that this method could be used for infants' facial expression recognition with good performance. Moreover, the presented method achieved low computation complexity, thus it is suitable for video surveillance applications such as intelligent baby-care.
\end{abstract}

Keywords Infants • Facial Expression Recognition (FER) • Face image database

\section{Introduction}

Man's infancy is very important in the process of personal growth. In recent years, the technology of video surveillance has developed rapidly. In addition to its traditional function, it has been added else functions like dynamic surveillance and

\footnotetext{
${ }^{1}$ Wenping Lu

School of Electronic Information Engineering, Tianjin University

e-mail: happyluren@163.com

Min Li

School of Electronic Information Engineering, Tianjin University

limin_0724@163.com

Wenfei Hao

School of Electronic Information Engineering, Tianjin University

haowenfei@tju.edu.cn

Yu Liu( $\square)$

School of Electronic Information Engineering, Tianjin University

liuyu@tju.edu.cn
} 
face recognition. But it is not enough. It becomes essential to nurse and monitor the infants' health intelligently by studying facial expression features.

After years of research, technology of FER has developed greatly. An important part of FER is extracting expression features, and many relatively mature algorithms have been proposed in the past few decades, such as the method based on geometric features and the method based on appearance. In the method based on geometric features, Panic extracted features from the front face and side face by using multiple detectors. But this method could be hardly used in real-life applications because it ignored some changes so that it could cause the poor differentiation [9], such as wrinkle and illumination. The method based on appearance mainly employed the partial feature of appearance. It filtered images through a group of filters, and its result could reflect the relationship between pixels. For example, Gabor wavelet can be used to extract facial features [7], [10].

However, the relative studies before all depend on adult face image database. This paper aims to build a FER method for infants, which can be used during video surveillance, and provides an infants' FER solution for low-cost embedded surveillance system by analyzing infant facial features of different expressions, especially eyes and mouth's open and close degree [6].

\section{Database of Infants Face Image}

There are more than a dozen of face databases to be chosen when analyzing facial features of adults, such as Richard's MIT database, The Yale Face Database, The Japanese Female Facial Expression (JAFFE) Database [2], [5] and so on. There are 82 male faces and 74 female faces in MIT face database, 6 face images per person. While Yale face database collects 165 male faces from 15 persons, 11 face images per person. JAFFE face database has 70 female faces from 10 persons, 7 face images per person, and it covers 7 expressions, such as normal, happy, sad, amazed, angry, disgusted and fearful [1].

Although adult face and infant face are similar in great degree, they are different. And faces will change greatly with age [8]. Infant face is soft, and its components are mostly fat so that some features which are obvious on adult's face will not appear clearly on the infant's face. The differences between infant face and adult face are that the proportion of infants' eyes is bigger, infant's face is more round and more lubrication, infant's eyebrows are short and flat, and his nose is relatively short and small [8]. Fig. 1 shows these different areas. Because of these differences, the FER methods based on adult face are not suitable for FER of infants. So an infant face image database is important and essential for analyzing face features of infants and recognizing facial expressions. 


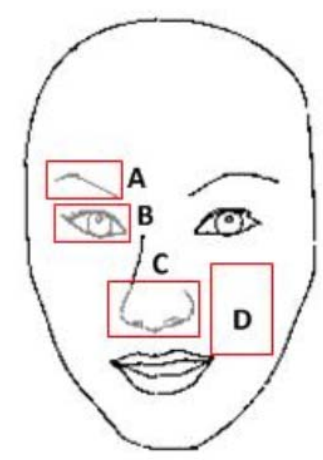

Fig. 1 Some different areas between infant face and adult faces

Unlike adult face image databases, collecting and building an infant face image database will cost much more. Firstly, there are few infant volunteers. Secondly, it is difficult to collect face images with different expressions of one infant. So this paper tries to build an infant face image database by an unconventional way. About 403 images including infant faces are downloaded. And an observing team of 10 people observe carefully and decides to divide infant facial expressions into four categories: crying, happy, sleepy and normal. As we know, infant's brain and psychology are not mature, and their facial muscles are just in developing stage, so the only way of communication for an infant is simple facial expressions. Moreover, infants can not show complex expressions such as sadness, angriness and disgust. Then we delete some images which include non-front faces, obscured faces or whose pixel is too low. At last, we get 129 infant faces, including 25 crying faces, 33 happy faces, 34 sleepy faces and 37 normal faces [4]. Then we pretreat these images before feature extraction, including the normalization of the size of the facial images and transformation into gray scale image. These infant face images are derived from Internet, and it is more universal than other face image databases made by conventional way, such as MIT adult face database.

\section{Face Images Analyzing and Processing}

We have given a simple analysis on features of infants' face above. Now we analyze features of infant faces from the perspective of image processing. 


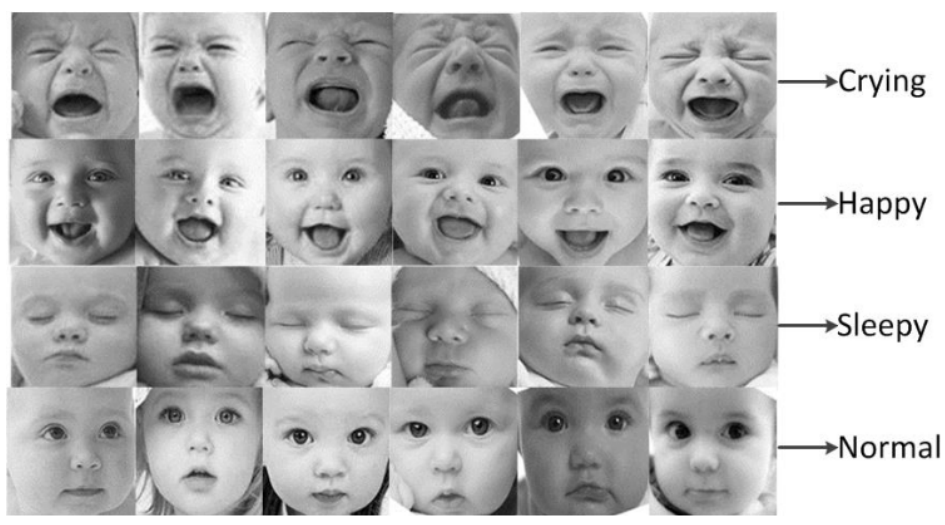

Fig. 2 Four categories of infant face images

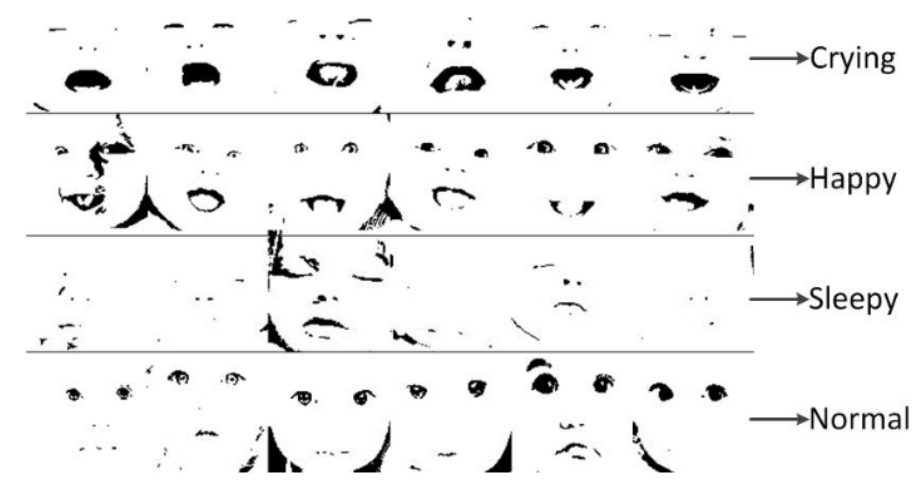

Fig. 3 Face images in Fig. 2 after binarization processing

Fig. 2 shows a part of gray-level images of the infant face from the database we built. We can recognize their expressions from top to bottom in Fig. 2: crying, happy, sleepy and normal. The gray value at coordinates $(\mathrm{x}, \mathrm{y})$ can be defined as $f(x, y)$. According to our subjective experience and decision, we know features of eyes and mouth have a higher recognition for facial expressions than other face features. Then we convert the gray image $\mathrm{f}(\mathrm{x}, \mathrm{y})$ into binary image $\mathrm{g}(\mathrm{x}, \mathrm{y})$.

$$
g(x, y)= \begin{cases}0, & f(x, y)=255 \\ 1, & f(x, y)=0\end{cases}
$$

As Fig. 3 shows, we can see that eyes area keeps few black pixels in crying face images after binarization processing, while mouth area keep a great number of black pixels. For face images with happy expression, we can see eyes area and mouth area both keep many black pixels.

Based on the above analysis on eyes and mouth features, this paper proposes a method for infants FER by judging the open and closed degree of infants' eyes and mouth. In order to reduce errors and amount of calculation, we employ ma- 
thematical statistical methods to get the area of eyes and mouth. Eyes area is located in a rectangular area, whose coordinates are $(10,18),(90,18),(10,42),(90$, 42), marked as Area_interested_e, while mouth area is also located in a rectangular area, whose coordinates are $(21,54),(77,54),(21,86),(77,86)$, marked as Area_interested_m. Fig. 4 shows that Area_interested_e and Area_interested_m cover eyes and mouth perfectly.
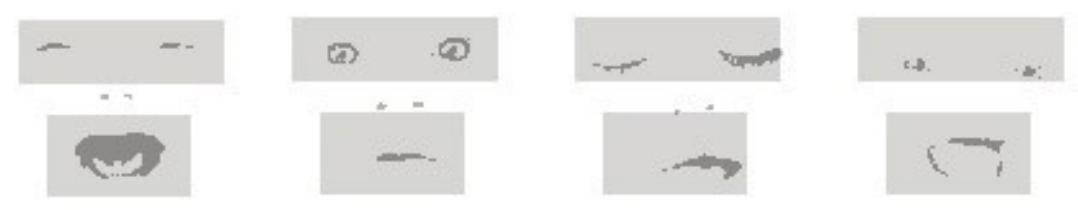

Fig. 4 Area_interested's coverage of eye and mouth

After finishing the binarization of infant face images, we calculate the sum of black pixels of eyes and mouth, which is used for representing area of eyes and mouth, marked as $S_{e}$ and $S_{m}$

$$
\begin{aligned}
& \mathrm{S}_{e}=\sum_{x=m_{1}}^{m_{2}} \sum_{y=n_{1}}^{n_{2}} g(x, y) \\
& \mathrm{S}_{m}=\sum_{x=p_{1}}^{p_{2}} \sum_{y=q_{1}}^{q_{2}} g(x, y)
\end{aligned}
$$

Where $\mathrm{m} 1, \mathrm{~m} 2, \mathrm{n} 1, \mathrm{n} 2, \mathrm{p} 1, \mathrm{p} 2, \mathrm{q} 1, \mathrm{q} 2$ are values corresponding to vertices' coordinates of rectangular area as above. In our data base, theses parameters are set as: $\mathrm{m} 1=10, \mathrm{~m} 2=90, \mathrm{n} 1=18, \mathrm{n} 2=42, \mathrm{p} 1=21, \mathrm{p} 2=77, \mathrm{q} 1=54, \mathrm{q} 2=86$. And the area difference between eyes area and mouth area can be defined as $\mathrm{D}$,

$$
D=S_{e}-S_{m}
$$

Because images in the database are derived from Internet, gray level of images differs from each other. When employing the binarization, this paper selects 70 as the binarization threshold which is got by statistical methods and reflects features of eyes and mouth well.

However, as eyes area and mouth area have high brightness in some face images, especially in crying and happy expression face images, these areas will lose some black pixels after binarization, which means the sum of black pixels calculated is much less than the real sum. To solve this problem, this paper works it out by employing the partial completion processing. During this processing, we scan the mouth area and judge it. If there are more than one black pixel in the same row, we make pixels between the ones black. Fig. $5 \mathrm{a}$ and Fig. 5b show the effect of partial completion processing for face images of crying and happy expressions. 


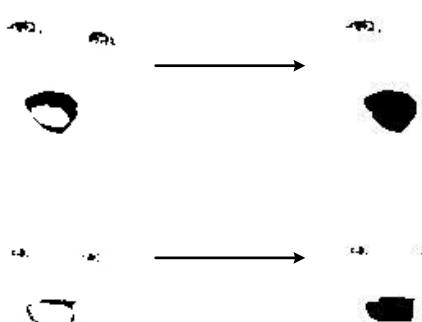

a

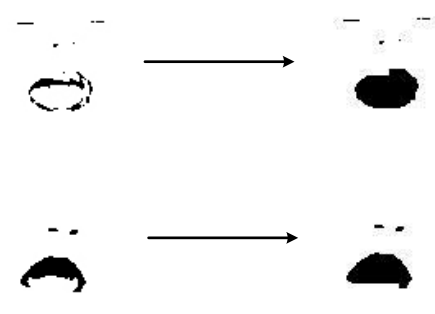

b

Fig. 5 The effect of partial completion processing for face images of crying and happy expressions

This paper randomly chooses 10 face images per facial expression from the database. Fig. 6 shows the performance of Se, Sm and D. In Fig. 6, the abscissa stands for the number of face images, and the ordinate stands for value of Se, Sm and D. The curve of symbol ' + ' is Sm, the curve of symbol ' '? is Se, and the curve of symbol ' $*$ ' is $\mathrm{D}$.
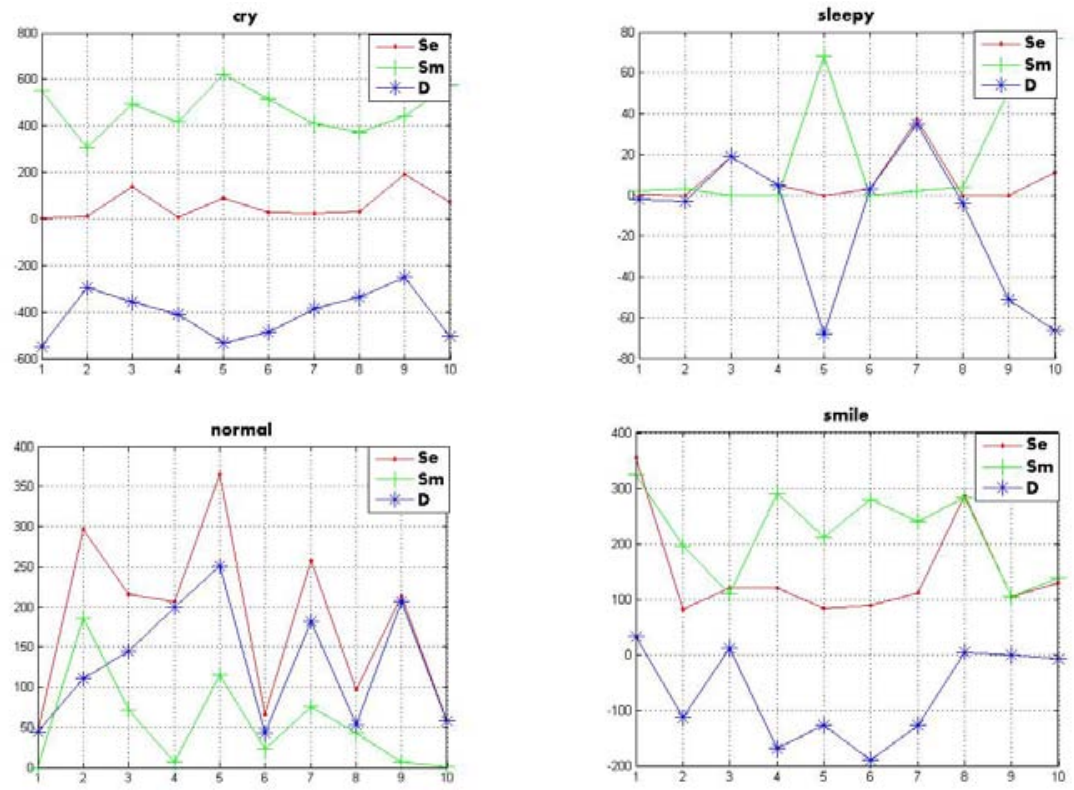

Fig. 6 The curves of Se, Sm and D in four different facial expressions 


\section{Infant FER}

Based on above analysis and experiment results, this paper obtains an algorithm for infant FER below:

Algorithm 1

1. Detect the infant face in the image and cut the region off the image, marked $I m$ (This paper mainly discusses the recognition method for the facial expression, so the methods for face detecting can be obtained from [3]).

2. Normalize the size of the facial image $I m$. Transform the $I m$ into $\operatorname{Im}_{100 * 100}$, which is $100 \times 100$ pixels by using the related function, such as "imresize()" in matlab.

3. Transform the $I m \_r e s i z e d$ into gray scale image $I m \_g r a y$.

4. Convert the Im_grayinto binary image and use partial completion processing.

5. Calculate the parameters: $S e, S m$ and $D$.

6. If ( $\mathrm{D}<=-200$ )

7. the facial expression is "Crying"

8. Elseif ( $\mathrm{Se}<=100$ \&\& $\mathrm{Sm}<=100$ \&\& $\mathrm{D}<=30$ \& \& $\mathrm{D}>=-70$ )

9. the facial expression is "Sleeping"

10. Elseif ( $\mathrm{D}>=0)$

11. the facial expression is "Normal"

12. Else

13. the facial expression is "Happy"

14. End

Based on the above algorithm, we do some experiments to verify its accuracy . The result is shown in Table 1.

As the experiment results show in Table 1, we implement infants FER based on the open and close degree of infant's eyes and mouth by employing the binarization and the threshold value. Running the matlab coding on the laptop platform, on which CPU is the Intel Core 2 Duo and the dominant frequency is $2.4 \mathrm{GHZ}$, the result shows that recognizing infant facial expression costs about 0.123 seconds per facial image whose size is $100 \times 100$ pixels. We conclude that the method proposed in the paper has the low algorithm complexity and can be transplanted to the embedded platform such as the TI DM365 embedded platform to complete the FER during surveillance the babies.

Table 1 Experiment results of FER on infants face image database

\begin{tabular}{cccc}
\hline Expressions & Num of Test & Num of accuracy & accuracy \\
\hline Crying & 15 & 15 & $100 \%$ \\
Sleepy & 24 & 22 & $91.7 \%$ \\
Normal & 23 & 21 & $91.3 \%$ \\
Happy & 27 & 25 & $92.6 \%$ \\
\hline
\end{tabular}




\section{Conclusions}

In this paper, an infant face image database is built by an untraditional way. To recognize face expressions of infants, this paper analyzes the open and close degree of eyes and mouths according to the database and proposes a method of FER by using three parameters, Se, Sm and D. Moreover, the proposed method can be applied on the embedded system platform. The experimental results show that the proposed method offers high accuracy of infant facial expression, and the amount of calculation and the time is not large.

\section{Acknowledgments}

This work was supported in part by the National Natural Science Foundation of China under Grant 61373102.

\section{Refer ences}

1. Ekman P, Friesen WV. Facial Action Coding System. A Technique for the Measurementof Facial Movement. Palo Alto:Consulting Psychologists Press, 1978.

2. Fei Cheng, Jiangsheng Yu, Huilin Xiong. Facial Expression Recognition in JAFFE Dataset Based on Gaussian Process Classification, Neural Networks, IEEE Transactions on . Publication Year: 2010, Page(s): 1685- 1690.

3. GUO Lei, WANG Qiu-guang. "Research of Face Detection Based on Adaboost Algorithm and OpenCV Implementation”, Journal of Harbin University of Science and Technology, 2009, 05.

4. http://pan.baidu.com/s/1ghTiB. Accessed 22 Oct 2013.

5. http://web.mit.edu/emeyers/www/face_databases.html. Accessed 22 Oct 2013.

6. Huang Mei, He Ningfeng, Zhang Yashu. Sleep monitoring of infants and very young children based on eye states recognition. Computer Applications and Software, vol.29 No.8 Aug.2012.

7. Jingfu Ye, Yongzhao Zhan, Shunlin Song. Facial expression features extraction based on Gabor wavelet transformation. 2004 IEEE International Conference on, vol.3, no., pp.2215,2219 vol.3, 10-13 Oct. 2004.

8. Marquardt Beauty Analysis, Inc. Face Variations By Age. http:// www.beautyanalysis.com/mba_facevariationsbyage_page.htm.

9.Pantic M, Rothkrantz L . Automatic analysis offacial expressions: the state of the art[J]. IEEE Transactions on Pattern Analysis and MachineIntelligence, 2000,22(12):14241445.

10. Xiaoli Li, Qiuqi Ruan, Chengxiong Ruan. Facial expression recognition with local Gabor filters. Signal Processing (ICSP), 2010 IEEE 10th International Conference on, vol., no., pp.1013,1016, 24-28 Oct. 2010. 